\title{
Preparation, characterization and thermal analysis of metal hydrazinocarboxylate derivatives
}

\author{
P RAVINDRANATHAN and K C PATIL* \\ Department of Inorganic and Physical Chemistry, Indian Institute of Science, \\ Bangalore 560012, India
}

MS received 12 December 1984

Abstract. The reaction of hydrazinocarboxylic acid, $\mathrm{N}_{2} \mathrm{H}_{3} \mathrm{COOH}$, with metal ions in the presence of hydrazine hydrate yields metal hydrazinocarboxylate derivaties like: $\mathrm{M}\left(\mathrm{N}_{2} \mathrm{H}_{3} \mathrm{COO}\right)_{2} \cdot n \mathrm{H}_{2} \mathrm{O}, \mathrm{M}=\mathrm{Mg}$, Ca and $\mathrm{Mn} ; \mathrm{M}\left(\mathrm{N}_{2} \mathrm{H}_{3} \mathrm{COO}\right)_{2}\left(\mathrm{~N}_{2} \mathrm{H}_{4}\right)_{2}, \mathrm{M}=\mathrm{Mn}, \mathrm{Fe}, \mathrm{Co}$, $\mathrm{Ni}$ and $\mathrm{Zn} ; \mathrm{N}_{2} \mathrm{H}_{5} \mathrm{M}\left(\mathrm{N}_{2} \mathrm{H}_{3} \mathrm{COO}\right)_{3} \cdot \mathrm{H}_{2} \mathrm{O}, \mathrm{M}=\mathrm{Fe}, \mathrm{Co}, \mathrm{Ni}, \mathrm{Zn}$; and $\mathrm{N}_{2} \mathrm{H}_{5} \mathrm{Mg}\left(\mathrm{N}_{2} \mathrm{H}_{3} \mathrm{COO}\right)_{3}$. Reaction conditions to obtain a desired product have been standardised and a probable reaction mechanism for the formation of different productsproposed. Thermal analysis of the compounds has been investigated using simultaneous to-DTG-DTA. Metal hydrazinocarboxylate derivatives decompose in air at low temperatures to the respective metal oxides.

Keywords. Metal hydrazinocarboxylales; infrared spectra; thermal analysis.

\section{Introduction}

Transition metal ions are known to react with hydrazine hydrate saturated with carbon dioxide to yield metal hydrazinocarboxylate derivatives like $\mathrm{M}\left(\mathrm{N}_{2} \mathrm{H}_{3} \mathrm{COO}\right)_{2}$, $\mathrm{M}\left(\mathrm{N}_{2} \mathrm{H}_{3} \mathrm{COO}\right)_{2} . n \mathrm{H}_{2} \mathrm{O}, \mathrm{M}\left(\mathrm{N}_{2} \mathrm{H}_{3} \mathrm{COO}\right)_{2}\left(\mathrm{~N}_{2} \mathrm{H}_{4}\right)_{2}$ or $\mathrm{N}_{2} \mathrm{H}_{5} \mathrm{M}\left(\mathrm{N}_{2} \mathrm{H}_{3} \mathrm{COO}\right)_{3} \mathrm{H}_{2} \mathrm{O}$. Hydrazinocarboxylic acid, $\mathrm{N}_{2} \mathrm{H}_{3} \mathrm{COOH}$ formed metathetically by the reaction of $\mathrm{CO}_{2}$ with $\mathrm{N}_{2} \mathrm{H}_{4}$ appears to react with the metal ions to form these complexes. Ferrari et $a l$ (1965)and Braibanti et al (1966, 1967a, b, 1968,1971)have reported the preparation, IR spectra and crystal structures of a number of these complexes. They have shown that the hydrazinocarboxylate anion, $\mathrm{N}_{2} \mathrm{H}_{3} \mathrm{COO}^{-}$acts as a bidentate group coordinating to the metal through both $\mathrm{N}$ and $\mathrm{O}$ atoms, Thermal decomposition of some metal hydrazinocarboxylate complexes has been studied and reported to yield finely divided metals or metal oxides (Macek et al 1976).

During the course of our studies on hydrazine derivatives we have reported the preparation and thermal properties of some metal hydrazinocarboxylate derivatives (Patil et al 1979a, b, 1983). They were prepared both by homogeneous and heterogeneous reactions. However, the exact reaction conditions to obtain a desired product do not appear to have been standardised. Present study is, therefore, aimed at standardising the reaction conditions to obtain any desired product and suggesting a possible reaction mechanism. Metal hydrazinocarboxylate derivatives are of interest as precursors to metal and metal oxides.

- To whom all correspondence should be addressed. 


\section{Experimental}

Hydrazine hydrate $\left(\mathrm{N}_{2} \mathrm{H}_{4} \cdot \mathrm{H}_{2} \mathrm{O}\right)$ 99-100\%, BDH, was used in all the reactions.

\subsection{Preparation of hydrazinocarboxylic acid, $\mathrm{N}_{2} \mathrm{H}_{3} \mathrm{COOH}$}

(a) Hydrazine hydrate was saturated with carbon dioxide at room temperature until a white precipitate separated. The precipitate was dried and stored over $\mathrm{P}_{2} \mathrm{O}_{5}$ in a vacuum desicator.

(b) Dissolution of $25 \mathrm{~g}$ of ammonium carbonate in $50 \mathrm{ml}$ of $\mathrm{N}_{2} \mathrm{H}_{4} \cdot \mathrm{H}_{2} \mathrm{O}$ gave a viscous liquid $\left(\mathrm{N}_{2} \mathrm{H}_{5} \mathrm{COON} \mathrm{H}_{3}\right)$. Passing $\mathrm{CO}_{2}(g)$ through this resulted in the formation of a white solid which was dried and stored over $\mathrm{P}_{2} \mathrm{O}_{3}$ in a vacuum desicator.

The chemical analysis and IR spectrum of the white solid correspond to that of $\mathrm{N}_{2} \mathrm{H}_{3} \mathrm{COOH}$. A $5 \%$ solution of $\mathrm{N}_{2} \mathrm{H}_{3} \mathrm{COOH}$ in $\mathrm{N}_{2} \mathrm{H}_{4} \cdot \mathrm{H}_{2} \mathrm{O}$ was used in the preparation of metal hydrazinocarboxylate hydrates and hydrazinates. In the case of hydraziniumderivativesa $15 \%$ solution of $\mathrm{N}_{2} \mathrm{H}_{3} \mathrm{COOH}$ in $\mathrm{N}_{2} \mathrm{H}_{4} \cdot \mathrm{H}_{2} \mathrm{O}$ was employed.

\subsection{Preparation of metal hydrazinocarboxylate hydrates and hydrazinates}

Anaqueoussolution containing metal ions like $\mathrm{Mg}^{2+}, \mathrm{Ca}^{2+}, \mathrm{Mn}^{2+}, \mathrm{Fe}^{2+}, \mathrm{Co}^{2+}, \mathrm{Ni}^{2+}$ or $\mathrm{Zn}^{2+}(25 \mathrm{ml}, 04 \mathrm{M})$. was treated with a $5 \%$ solution of $\mathrm{N}_{2} \mathrm{H}_{3} \mathrm{COOH}$ in $\mathrm{N}_{2} \mathrm{H}_{4} \cdot \mathrm{H}_{2} \mathrm{O}$ until the precipitateformed initiallyjust dissolved.The clear solution thus obtained was kept open to the atmosphere. Crystalline solids separated from the solution within a couple of days. The composition of the crystals was fixed by chemical analysis to be $\mathrm{M}\left(\mathrm{N}_{2} \mathrm{H}_{3} \mathrm{COO}\right)_{2} \cdot n \mathrm{H}_{2} \mathrm{O}, \mathrm{M}=\mathrm{Mg}, \mathrm{Ca}$ and $\mathrm{Mn}$; and $\mathrm{M}\left(\mathrm{N}_{2} \mathrm{H}_{3} \mathrm{COO}\right)_{2}\left(\mathrm{~N}_{2} \mathrm{H}_{4}\right)_{2}, \mathrm{M}$ $=\mathrm{Mn}, \mathrm{Fe}, \mathrm{Co}, \mathrm{Ni}$ and $\mathrm{Zn}$ (table 1). Manganese hydrazinocarboxylate hydrazinate, $\mathrm{Mn}\left(\mathrm{N}_{2} \mathrm{H}_{3} \mathrm{COO}\right)_{2}\left(\mathrm{~N}_{2} \mathrm{H}_{4}\right)_{2}$ was obtained by treating a fine powder of $\mathrm{Mn}\left(\mathrm{N}_{2} \mathrm{H}_{3} \mathrm{COO}\right)_{2} \cdot 2 \mathrm{H}_{2} \mathrm{O}$ with $\mathrm{N}_{2} \mathrm{H}_{4} \cdot \mathrm{H}_{2} \mathrm{O}$.

\subsection{Preparation of hydrazinium metal hydrazinocarboxylate derioatioes}

An aqueous solution containing metal ions like $\mathrm{Fe}^{2+}, \mathrm{Co}^{2+}, \mathrm{Ni}^{2+}$ or $\mathrm{Zn}^{2+}(25 \mathrm{ml}$, $04 \mathrm{M}$ ) was treated with a $15 \%$ solution of $\mathrm{N}_{2} \mathrm{H}_{3} \mathrm{COOH}$ in $\mathrm{N}_{2} \mathrm{H}_{4} \cdot \mathrm{H}_{2} \mathrm{O}$ until the precipitate formed initially just dissolved. The resulting clear solution was left open to atmosphere. Beautiful crystals separated from the solution in 3-4 days. The composition of the crystals was fixed by chemical analysis to be $\mathrm{N}_{2} \mathrm{H}_{5} \mathrm{M}\left(\mathrm{N}_{2} \mathrm{H}_{3} \mathrm{COO}\right)_{3} \cdot \mathrm{H}_{2} \mathrm{O}, \mathrm{M}=\mathrm{Fe}, \mathrm{Co}, \mathrm{Ni}$ and $\mathrm{Zn}$ (table 1).

Hydrazinium magnesium hydrazinocarboxylate, $\mathrm{N}_{2} \mathrm{H}_{5} \mathrm{Mg}\left(\mathrm{N}_{2} \mathrm{H}_{3} \mathrm{COO}\right)_{3}$ was obtained by dissolving magnesium powder in the $15 \%$ solution of $\mathrm{N}_{2} \mathrm{H}_{3} \mathrm{COOH}$ in $\mathrm{N}_{2} \mathrm{H}_{4} \cdot \mathrm{H}_{2} \mathrm{O}$. The complex was precipitated by the addition of alcohol.

\subsection{Analysis}

The metal content in the complexes were determined by EDTA complexometric titration. Hydrazine content was estimated volumetrically using $0.025 \mathrm{M} \mathrm{KIO}_{3}$ solution under Andrews' conditions (Vogel 1961).

\subsection{Physicochemical studies}

Infrared spectra of the complexes were recorded as nujol mulls using a Perkin Elmer 781 Spectrophotometer. Simultaneous TG-DTG-DTA of the samples were recorded usinga 
Table 1. Chemical analysis of metal hydrazinocarboxylate derivatives.

\begin{tabular}{|c|c|c|c|c|}
\hline \multirow[b]{2}{*}{ Compound } & \multicolumn{2}{|c|}{$\%$ of Metal } & \multicolumn{2}{|c|}{$\%$ of Hydrazine } \\
\hline & Observed & Required & Observed & Required \\
\hline $\mathrm{Mg}\left(\mathrm{N}_{2} \mathrm{H}_{3} \mathrm{COO}\right)_{2}\left(\mathrm{H}_{2} \mathrm{O}\right)_{2}$ & 11.44 & 11.55 & 3024 & 3043 \\
\hline $\mathrm{Ca}\left(\mathrm{N}_{2} \mathrm{H}_{3} \mathrm{COO}\right)_{2} \mathrm{H}_{2} \mathrm{O}$ & $19 \cdot 12$ & 19.26 & 3064 & 3075 \\
\hline $\mathrm{Mn}\left(\mathrm{N}_{2} \mathrm{H}_{3} \mathrm{COO}\right)_{2}\left(\mathrm{H}_{2} \mathrm{O}\right)_{2}$ & $22: 75$ & $22 \cdot 80$ & 2646 & 26.56 \\
\hline $\mathrm{Mn}\left(\mathrm{N}_{2} \mathrm{H}_{3} \mathrm{COO}\right)_{2}\left(\mathrm{~N}_{2} \mathrm{H}_{4}\right)_{2}$ & 2022 & 2042 & 4732 & 47.59 \\
\hline $\mathrm{Fe}\left(\mathrm{N}_{2} \mathrm{H}_{3} \mathrm{COO}\right)_{2}\left(\mathrm{~N}_{2} \mathrm{H}_{4}\right)_{2}$ & 20.70 & 2069 & 47.50 & 47.47 \\
\hline $\mathrm{Co}\left(\mathrm{N}_{2} \mathrm{H}_{3} \mathrm{COO}\right)_{2}\left(\mathrm{~N}_{2} \mathrm{H}_{4}\right)_{2}$ & 21.60 & 21.58 & 46.40 & $46 \cdot 89$ \\
\hline $\mathrm{Ni}\left(\mathrm{N}_{2} \mathrm{H}_{3} \mathrm{COO}\right)_{2}\left(\mathrm{~N}_{2} \mathrm{H}_{4}\right)_{2}$ & 21.48 & 21.52 & $46 \cdot 23$ & 4693 \\
\hline $\mathrm{Zn}\left(\mathrm{N}_{2} \mathrm{H}_{3} \mathrm{COO}\right)_{2}\left(\mathrm{~N}_{2} \mathrm{H}_{4}\right)_{2}$ & 23.23 & 23.39 & 45.38 & $45 \cdot 81$ \\
\hline $\mathrm{N}_{2} \mathrm{H}_{3} \mathrm{Mg}\left(\mathrm{N}_{2} \mathrm{H}_{3} \mathrm{COO}\right)_{3}$ & 7.99 & 8.01 & 43.00 & 42.62 \\
\hline $\mathrm{N}_{2} \mathrm{H}_{5} \mathrm{Fe}\left(\mathrm{N}_{2} \mathrm{H}_{3} \mathrm{COO}\right)_{3} \cdot \mathrm{H}_{2} \mathrm{O}$ & $16 \cdot 32$ & $16 \cdot 82$ & $38 \cdot 64$ & 38.57 \\
\hline $\mathrm{N}_{2} \mathrm{H}_{5} \mathrm{Co}\left(\mathrm{N}_{2} \mathrm{H}_{3} \mathrm{COO}\right)_{3} \cdot \mathrm{H}_{2} \mathrm{O}$ & 1748 & 17.59 & $38 \cdot 11$ & 38.21 \\
\hline $\mathrm{N}_{2} \mathrm{H}_{5} \mathrm{Ni}\left(\mathrm{N}_{2} \mathrm{H}_{3} \mathrm{COO}\right)_{3} \cdot \mathrm{H}_{2} \mathrm{O}$ & 17.50 & 17.58 & 38.20 & 38.28 \\
\hline $\mathrm{N}_{2} \mathrm{H}_{5} \mathrm{Zn}\left(\mathrm{N}_{2} \mathrm{H}_{3} \mathrm{COO}\right)_{3} \cdot \mathrm{H}_{2} \mathrm{O}$ & 19.08 & 19.14 & 37.56 & 37.49 \\
\hline
\end{tabular}

TGD-5000 RH. thermobalance of ULVAC-RIKO, Japan. All the experiments were carried out using $45 \mathrm{mg}$ samples under ambient conditions. The heating rate employed was $20^{\circ} \mathrm{C} / \mathrm{min}$. The decomposition/combustion residues of the complexes were characterized by $\mathrm{x}$-ray powder diffraction patterns recorded on a Philips $\mathrm{PW}$ 1050/70 diffractometer using $\mathrm{CuK} \alpha$ and $\mathrm{CoK} \alpha$ radiation.

\section{Results and discussion}

\subsection{Hydrazinocarboxylic acid, $\mathrm{N}_{2} \mathrm{H}_{3} \mathrm{COOH}$}

Stolle and Hoffman (1904)have reported the formation of hydrazinocarboxylic acid, $\mathrm{N}_{2} \mathrm{H}_{3} \mathrm{COOH}$ by passing a current of $\mathrm{CO}_{2}$ through a well cooled solution of hydrazine. The acid, $\mathrm{N}_{2} \mathrm{H}_{3} \mathrm{COOH}$, was reported to decompose partially with the evolution of $\mathrm{CO}$, when heated $\left(\sim 90^{\circ} \mathrm{C}\right)$ to give a viscous liquid whose composition was not exactly known. Presently, we were able to prepare $\mathrm{N}_{2} \mathrm{H}_{3} \mathrm{COOH}$ not only by passing a current of $\mathrm{CO}_{2}$ through $\mathrm{N}_{2} \mathrm{H}_{4} \cdot \mathrm{H}_{2} \mathrm{O}$ at room temperature but also by the reaction of $\mathrm{CO}$, with $\mathrm{N}_{2} \mathrm{H}_{5} \mathrm{COON}_{2} \mathrm{H}_{3}$ prepared by dissolving ammonium carbonate in $\mathrm{N}_{2} \mathrm{H}_{4} \cdot \mathrm{H}_{2} \mathrm{O}$ (Patil et al 1979b). Thus the following equilibrium appears to exist in the $\mathrm{N}_{2} \mathrm{H}_{4} \cdot \mathrm{H}_{2} \mathrm{O} \cdot \mathrm{CO}_{2}$ reaction.

$$
\begin{aligned}
& \mathrm{N}_{2} \mathrm{H}_{4} \cdot \mathrm{H}_{2} \mathrm{O}+\mathrm{CO}_{2} \rightleftharpoons \mathrm{N}_{2} \mathrm{H}_{3} \mathrm{COOH} \mathbf{t} \mathrm{H}_{2} \mathrm{O} \\
& \text { (l) (g) (s) (l) } \\
& 2 \mathrm{~N}_{2} \mathrm{H}_{3} \mathrm{COOH} \rightleftharpoons \mathrm{N}_{2} \mathrm{H}_{5} \mathrm{COON} \mathrm{N}_{2} \mathrm{H}_{3}+\mathrm{CO}_{2} \text {. } \\
& \text { (s) (l) (g) }
\end{aligned}
$$

Hydrazinocarboxylicacid (carbazic acid) is highly hygroscopic and should be stored over $\mathrm{P}_{2} \mathrm{O}_{3}$ or $\mathrm{H}_{2} \mathrm{SO}_{4}$ in a vacuum desicator. It dissolves in water with dissociation as shown in (1). The acid was characterized by chemical analysis $\left(\% \mathrm{~N}_{2} \mathrm{H}_{4}\right.$-observed 44.55. required 44.74) and infrared spectra (figure 1). Characteristic infrared absorption bands of $\mathrm{N}_{2} \mathrm{H}_{3} \mathrm{COO}^{-}$group are seen at 3220,1650,1545, 1255, 1110, 995, 820, 770 and 


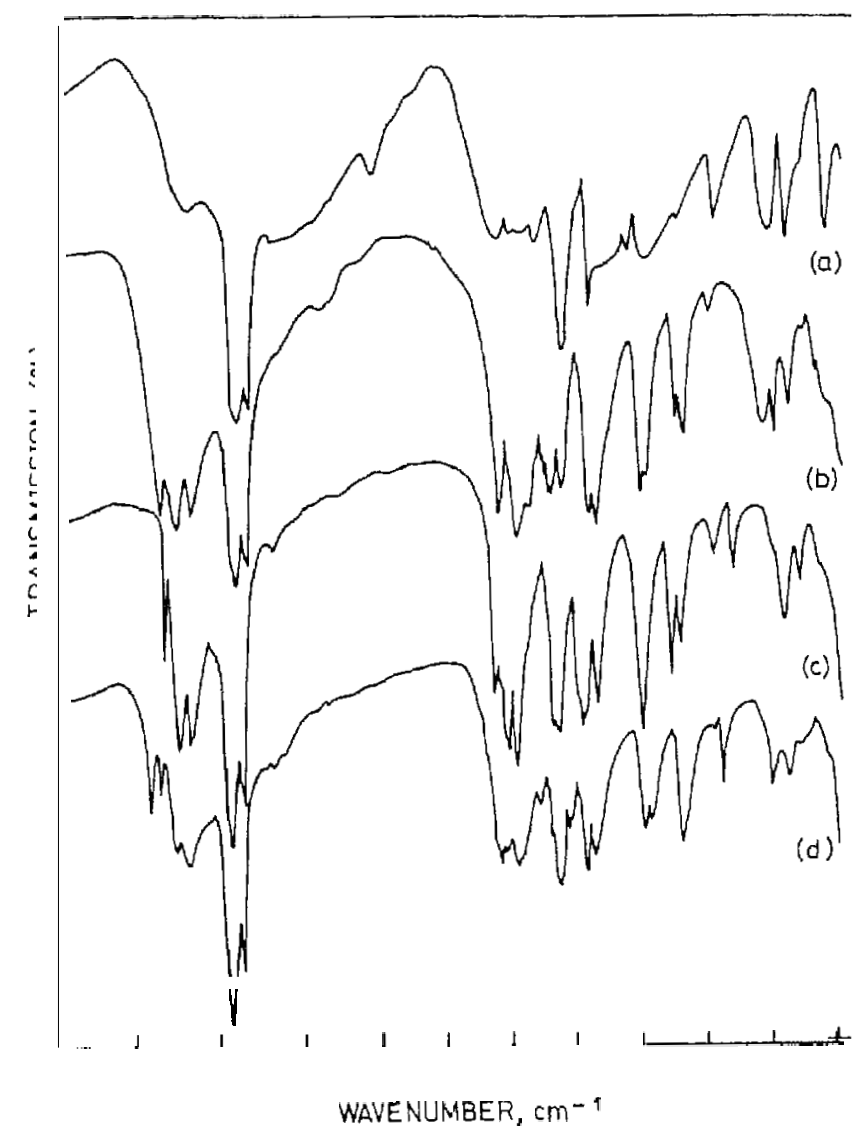

Figure 1. Infrared absorption spectra of (a) $\mathrm{N}_{2} \mathrm{H}_{3} \mathrm{COOH}$, (b) $\mathrm{Mg}\left(\mathrm{N}_{2} \mathrm{H}_{3} \mathrm{COO}\right)_{2}\left(\mathrm{H}_{2} \mathrm{O}\right)_{2}$, (c) $\mathrm{Co}\left(\mathrm{N}_{2} \mathrm{H}_{3} \mathrm{COO}\right)_{2}\left(\mathrm{~N}_{2} \mathrm{H}_{4}\right)_{2}$, (d) $\mathrm{N}_{2} \mathrm{H}_{5} \mathrm{Fe}\left(\mathrm{N}_{2} \mathrm{H}_{3} \mathrm{COO}\right)_{3} \cdot \mathrm{H}_{2} \mathrm{O}$.

$640 \mathrm{~cm}^{-1}$. The TG-DTG-DTA curve of $\mathrm{N}_{2} \mathrm{H}_{3} \mathrm{COOH}$ (figure 2) shows a two-step decomposition. The first step with a weight loss of $15 \%$ could be attributed to the partial decomposition of $\mathrm{N}_{2} \mathrm{H}_{3} \mathrm{COOH}$ to $\mathrm{N}_{2} \mathrm{H}_{5} \mathrm{COON}{ }_{2} \mathrm{H}_{3}$ with the evolution of $\mathrm{CO}_{2}$ as in (2). The corresponding reaction in DTA is seen as a broad endotherm at $107^{\circ} \mathrm{C}$ probably due to the combination of melting and decomposition. In the second step (100\% weight loss) the resulting viscous liquid $\mathrm{N}_{2} \mathrm{H}_{3} \mathrm{COON}_{2} \mathrm{H}_{3}$ volatalizes with decomposition.

\subsection{Mechanism}

The reaction of metal ions with $\mathrm{N}_{2} \mathrm{H}_{3} \mathrm{COOH}$ in $\mathrm{N}_{2} \mathrm{H}_{4} \cdot \mathrm{H}_{2} \mathrm{O}$ gave $\mathrm{M}\left(\mathrm{N}_{2} \mathrm{H}_{3} \mathrm{COO}\right)_{2}$. $n \mathrm{H}_{2} \mathrm{O}, \mathrm{M}\left(\mathrm{N}_{2} \mathrm{H}_{3} \mathrm{COO}\right)_{2}\left(\mathrm{~N}_{2} \mathrm{H}_{4}\right)_{2}$ and /or $\mathrm{N}_{2} \mathrm{H}_{5} \mathrm{M}\left(\mathrm{N}_{2} \mathrm{H}_{3} \mathrm{COO}\right)_{3} \cdot \mathrm{H}_{2} \mathrm{O}$ typecomplexes. It is interesting to note that dilute solutions of $\mathrm{N}_{2} \mathrm{H}_{3} \mathrm{COOH}$ in $\mathrm{N}_{2} \mathrm{H}_{4} \cdot \mathrm{H}_{2} \mathrm{O}$ gave either hydrates or hydrazinates and concentrated solutions $(15 \%)$ gave hydrazinium complexes. Since the solution of $\mathrm{N}_{2} \mathrm{H}_{3} \mathrm{COOH}$ in $\mathrm{N}_{2} \mathrm{H}_{4} \cdot \mathrm{H}_{2} \mathrm{O}$ essentially contains $\mathrm{N}_{2} \mathrm{H}_{5}^{+}, \mathrm{N}_{2} \mathrm{H}_{3} \mathrm{COO}^{-}, \mathrm{N}_{2} \mathrm{H}_{4} \cdot \mathrm{H}_{2} \mathrm{O}$ and $\mathrm{H}^{+}$species, one can 'write the following stoichiometric reactions for the various complexes formed. 


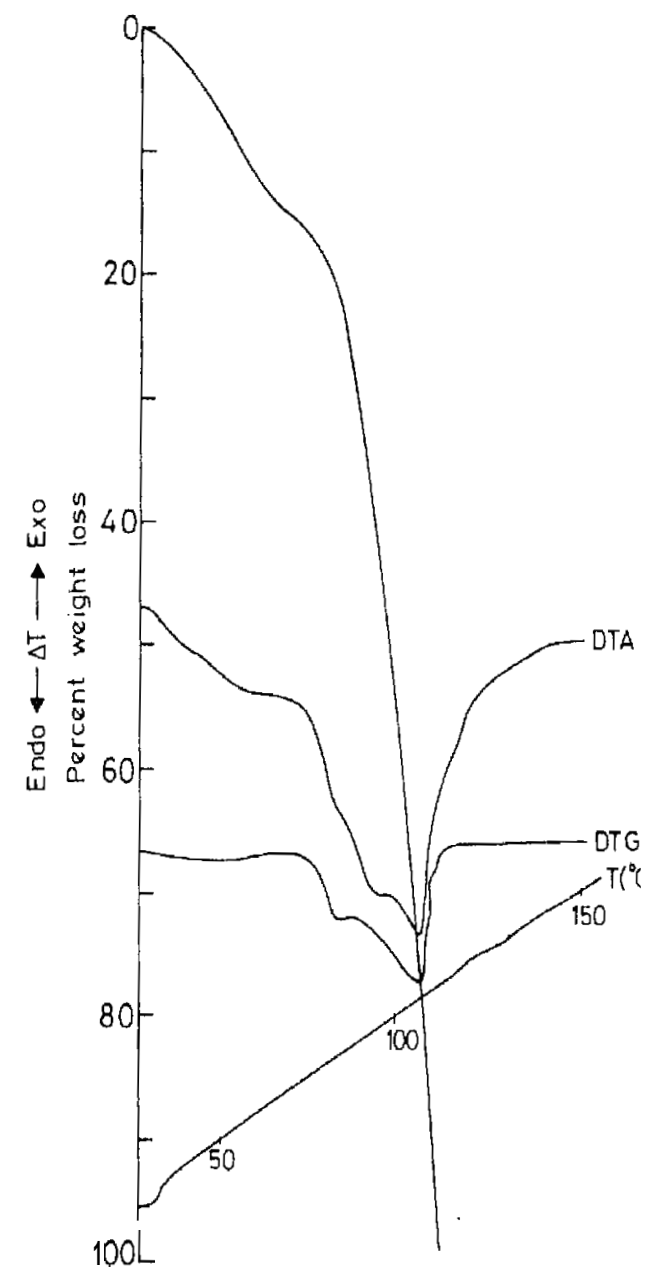

Figure 2. Simultaneous TG-DTG-DTA of $\mathrm{N}_{2} \mathrm{H}_{3} \mathrm{COOH}$.

$\mathrm{N}_{2} \mathrm{H}_{3} \mathrm{COOH} \mathbf{t} \mathrm{N}_{2} \mathrm{H}_{4} \cdot \mathrm{H}_{2} \mathrm{O} \rightarrow \mathrm{N}_{2} \mathrm{H}_{5} \mathrm{COON}_{2} \mathrm{H}_{3}+\mathrm{H}_{2} \mathrm{O}$

$\mathrm{MX}_{2}+2 \mathrm{~N}_{2} \mathrm{H}_{5} \mathrm{COON}_{2} \mathrm{H}_{3}+2 \mathrm{H}_{2} \mathrm{O} \rightarrow \mathrm{M}\left(\mathrm{N}_{2} \mathrm{H}_{3} \mathrm{COO}\right)_{2} \cdot 2 \mathrm{H}_{2} \mathrm{O}+2 \mathrm{~N}_{2} \mathrm{H}_{5} \mathrm{X}(4)$

$\mathrm{M}=\mathrm{Mg}, \mathrm{Ca}$ and $\mathrm{Mn}$.

$\mathrm{MX}_{2} \mathbf{t} 2 \mathrm{~N}_{2} \mathrm{H}_{5} \mathrm{COON}{ }_{2} \mathrm{H}_{3}+2 \mathrm{~N}_{2} \mathrm{H}_{4} \cdot \mathrm{H} 20 \rightarrow \mathrm{M}\left(\mathrm{N}_{2} \mathrm{H}_{3} \mathrm{COO}\right)_{2}\left(\mathrm{~N}_{2} \mathrm{H}_{4}\right)_{2}+$ $+2 \mathrm{~N}_{2} \mathrm{H}_{5} \mathrm{X}+2 \mathrm{H}_{2} \mathrm{O}$

$\mathrm{M}=\mathrm{Fe}, \mathrm{Co}, \mathrm{Ni}$ and $\mathrm{Zn}$.

$\mathrm{MX}_{2}+3 \mathrm{~N}_{2} \mathrm{H}_{5} \mathrm{COON}_{2} \mathrm{H}_{3} \mathbf{t} 3 \mathrm{H}_{2} \mathrm{O} \rightarrow \mathrm{N}_{2} \mathrm{H}_{5} \mathrm{M}\left(\mathrm{N}_{2} \mathrm{H}_{3} \mathrm{COO}\right)_{3} \cdot \mathrm{H}_{2} \mathrm{O}$ t $\mathbf{t} 2 \mathrm{~N}_{2} \mathrm{H}_{5} \mathrm{X}$

$\mathrm{M}=\mathrm{Fe}, \mathbf{C o}, \mathbf{N i}$ and $\mathrm{Zn}$. 
Thus, metal ions react with a dilute solution of $\mathbf{N}_{2} \mathrm{H}_{3} \mathbf{C O O H}$ in $\mathbf{N}_{\mathbf{2}} \mathrm{H}_{\mathbf{4}} \cdot \mathrm{H}_{2} \mathrm{O}$ to yield either $\mathrm{M}\left(\mathrm{N}_{2} \mathrm{H}_{3} \mathrm{COO}\right)_{2} \cdot 2 \mathrm{H}_{2} \mathrm{O}$ or $\mathrm{M}\left(\mathrm{N}_{2} \mathrm{H}_{3} \mathrm{COO}\right)_{2}\left(\mathrm{~N}_{2} \mathrm{H}_{4}\right)_{2}$. Only transition metal ions appear to form both hydrazinate and hydrazinium complexes. Hydrazinate complexes initially formed appear to react with excess of ligand (in concentrated solutions) to yield the corresponding hydrazinium salts as shown below:

$$
\begin{array}{r}
\mathrm{M}\left(\mathrm{N}_{2} \mathrm{H}_{3} \mathrm{COO}\right)_{2}\left(\mathrm{~N}_{2} \mathrm{H}_{4}\right)_{2}+\mathrm{N}_{2} \mathrm{H}_{5} \mathrm{COON}_{2} \mathrm{H}_{3}+3 \mathrm{H}_{2} \mathrm{O} \rightarrow \\
\mathrm{N}_{2} \mathrm{H}_{5} \mathrm{M}\left(\mathrm{N}_{2} \mathrm{H}_{3} \mathrm{COO}\right)_{3} \cdot \mathrm{H}_{2} \mathrm{O}+2 \mathrm{~N}_{2} \mathrm{H}_{4} \cdot \mathrm{H}_{2} \mathrm{O}
\end{array}
$$

This fact was confirmed by treating hydrazinate complexes with the ligand. However, manganese does not form $\mathrm{N}_{2} \mathrm{H}_{5} \mathrm{Mn}\left(\mathrm{N}_{2} \mathrm{H}_{3} \mathrm{COO}\right)_{3} \cdot \mathrm{H}_{2} \mathrm{O}$. The calcium salt appears to be unique in that it forms only monohydrate, $\mathrm{Ca}\left(\mathrm{N}_{2} \mathrm{H}_{3} \mathrm{COO}\right)_{2} \cdot \mathrm{H}_{2} \mathrm{O}$ whereas magnesium forms $\mathrm{N}_{2} \mathrm{H}_{5} \mathrm{Mg}\left(\mathrm{N}_{2} \mathrm{H}_{3} \mathrm{COO}\right)_{3}$ by the dissolution of the metal.

\subsection{Infrared spectra}

Infrared spectra of metal hydrazinocarboxylates have been studied and important IR absorption frequencies assigned (Patil et al 1983; Braibanti et al 1968). Typical IR spectra of $\mathrm{M}\left(\mathrm{N}_{2} \mathrm{H}_{3} \mathrm{COO}\right)_{2} \cdot 2 \mathrm{H}_{2} \mathrm{O}, \mathbf{M}\left(\mathrm{N}_{2} \mathrm{H}_{3} \mathrm{COO}\right)_{2}\left(\mathrm{~N}_{2} \mathrm{H}_{4}\right)_{2}$ and $\mathrm{N}_{2} \mathrm{H}_{5} \mathrm{M}\left(\mathrm{N}_{2} \mathrm{H}_{3} \mathrm{COO}\right)_{3} \cdot \mathrm{H}_{2} \mathrm{O}$ are shown in figure 1. As mentioned earlier, the $\mathrm{N}_{2} \mathrm{H}_{3} \mathrm{COO}^{-}$group acts as a bidentate ligand and coordinates to the metal through both the $\mathrm{N}$ and the $\mathrm{O}$ atoms forming a 5-membered ring. The characteristic $\mathrm{N}-\mathrm{N}$ stretching frequency of bidentate $\mathrm{N}_{2} \mathrm{H}_{3} \mathrm{COO}^{-}$is seen in the region 990-1015 $\mathrm{cm}^{-1}$ in all the complexes. The metal ions in all these complexes have octahedral coordination. The octahedra is completed by the two water molecules in $\mathrm{M}\left(\mathrm{N}_{2} \mathrm{H}_{3} \mathrm{COO}\right)_{2} \cdot 2 \mathrm{H}_{2} \mathrm{O}$; and by the two monodentate $\mathrm{N}_{2} \mathrm{H}_{4}$ groups in $\mathrm{M}\left(\mathrm{N}_{2} \mathrm{H}_{3} \mathrm{COO}\right)_{2}\left(\mathrm{~N}_{2} \mathrm{H}_{4}\right)$. Infrared spectra of the hydrazinates clearly show $v_{\mathrm{N}-\mathrm{N}}$ of monodentate $\mathrm{N}_{2} \mathrm{H}_{4} \sim 925 \mathrm{~cm}^{-1}$ in addition to $v_{\mathrm{N}-\mathrm{N}}$ of $\mathrm{N}_{2} \mathrm{H}_{3} \mathrm{COO}^{-} \sim 1000 \mathrm{~cm}^{-1}$. In the case of hydrazinium complexes, $\mathrm{N}_{2} \mathrm{H}_{5} \mathrm{M}\left(\mathrm{N}_{2} \mathrm{H}_{3} \mathrm{COO}\right)_{3} \cdot \mathrm{H}_{2} \mathrm{O}$ the octahedra around the metal atom involve 3 bidentate $\mathbf{N}_{2} \mathbf{H}_{3} \mathbf{C O O}^{-}$groups, neither $\mathrm{N}_{2} \mathrm{H}_{5}^{+}$nor $\mathrm{H}_{2} \mathrm{O}$ is coordinated to the metal. The characteristic IR frequency of ionic $\mathrm{N}_{2} \mathrm{H}_{5}^{+}$is seen $\sim 965 \mathrm{~cm}^{-1}$ in all these complexes.

\subsection{Thermal analysis}

The results of TG-DTG-DTA of $\mathrm{M}\left(\mathrm{N}_{2} \mathrm{H}_{3} \mathrm{COO}\right)_{2} n \mathrm{H}_{2} \mathrm{O}, \mathrm{M}\left(\mathrm{N}_{2} \mathrm{H}_{3} \mathrm{COO}\right)_{2}\left(\mathrm{~N}_{2} \mathrm{H}_{4}\right)_{2}$ and $\mathrm{N}_{2} \mathrm{H}_{5} \mathrm{M}\left(\mathrm{N}_{2} \mathrm{H}_{3} \mathrm{COO}\right)_{3} \cdot \mathrm{H}_{2} \mathrm{O}$ are summarized in tables $\mathbf{2 , 3}$ and $\mathbf{4}$ respectively. Some representative thermograms are shown in figure 3-5.

3.4a Metal hydrazinocarboxylate hydrates: All the metal hydrazinocarboxylate hydrates $\left[\mathbf{M}\left(\mathbf{N}_{2} \mathbf{H}_{3} \mathbf{C O O}\right)_{2} \cdot \boldsymbol{n} \mathbf{H}_{2} \mathrm{O}, \mathbf{M}=\mathrm{Mg}, \mathrm{Ca}\right.$ and $\left.\mathbf{M n}\right]$ lose the water of hydration initially to yield the corresponding metal hydrazinocarboxylates. The dehydration takes place at fairly high temperature $\left(100-200^{\circ} \mathrm{C}\right)$ indicating the presence of coordinated water molecules. The anhydrous metal hydrazinocarboxylates decompose exothermically to yield the corresponding metal oxides, The decomposition of the calcium salt shows 4 distinct steps corresponding to the formation of $\mathrm{Ca}\left(\mathrm{N}_{2} \mathrm{H}_{3} \mathrm{COO}\right)_{2}$, $\mathrm{CaC}_{2} \mathrm{O}_{4}, \mathrm{CaCO}_{3}$ and $\mathrm{CaO}$ respectively. Based on this observation and our early study (Patil et al 1979b) the intermediates in the decomposition of both $\mathrm{Mg}\left(\mathrm{N}_{2} \mathrm{H}_{3} \mathrm{COO}\right)_{2}$ and $\mathrm{Mn}\left(\mathrm{N}_{2} \mathrm{H}_{3} \mathrm{COO}\right)_{2}$ could be the corresponding metal oxalates. The composition of 
Table 2 Thermal analysis of metal hydrazinocarboxylate hydrate.

\begin{tabular}{|c|c|c|c|c|c|c|}
\hline \multirow[b]{3}{*}{ Compound } & \multirow{3}{*}{$\begin{array}{l}\text { DTA peak } \\
\text { temperature }\left({ }^{\circ} \mathrm{C}\right)\end{array}$} & \multirow{3}{*}{$\begin{array}{c}\text { Step } \\
\text { no. }\end{array}$} & \multicolumn{3}{|c|}{ Thermogravimetry } & \multirow[b]{3}{*}{ Products } \\
\hline & & & \multirow{2}{*}{$\begin{array}{l}\text { Temperature } \\
\text { range ("C) }\end{array}$} & \multicolumn{2}{|c|}{ Total weight loss (\%) } & \\
\hline & & & & Observed & Required & \\
\hline \multirow[t]{2}{*}{$\overline{\mathrm{Mg}\left(\mathrm{N}_{2} \mathrm{H}_{5} \mathrm{COO}\right)_{2}\left(\mathrm{H}_{2} \mathrm{O}\right)_{2}}$} & 170 (endo) & 1 & $150-180$ & $18 \cdot 00$ & 17.11 & $\mathrm{Mg}\left(\mathrm{N}_{2} \mathrm{H}_{3} \mathrm{COO}\right)_{2}$ \\
\hline & $\left.\begin{array}{l}325 \text { (exo) } \\
380 \text { (exo) }\end{array}\right\}$ & 2 & $180-485$ & $81 \cdot 00$ & 8083 & $\mathrm{MgO}$ \\
\hline \multirow[t]{4}{*}{$\mathrm{Ca}\left(\mathrm{N}_{2} \mathrm{H}_{3} \mathrm{COO}\right)_{2} \mathrm{H}_{2} \mathrm{O}$} & 135 (endo) & 1 & $115-152$ & 8.60 & 8.65 & $\mathrm{Ca}\left(\mathrm{N}_{2} \mathrm{H}_{3} \mathrm{COO}\right)_{2}$ \\
\hline & $\left.\begin{array}{l}281(\text { exo) } \\
308(\text { exo) }\end{array}\right\}$ & 2 & $152-310$ & 38.20 & $38-44$ & $\mathrm{CaC}_{2} \mathrm{O}_{4}$ \\
\hline & $\left.\begin{array}{l}345(\mathrm{exo}) \\
420(\mathrm{exo})\end{array}\right\}$ & 3 & $310-700$ & 51.80 & 51.90 & $\mathrm{CaCO}_{3}$ \\
\hline & 720 (endo) & 4 & $700-930$ & 73.00 & $73-04$ & $\mathrm{CaO}$ \\
\hline \multirow{3}{*}{$\mathrm{Mn}\left(\mathrm{N}_{2} \mathrm{H}_{3} \mathrm{COO}\right)_{2}\left(\mathrm{H}_{2} \mathrm{O}\right)_{2}$} & 130 (endo) & 1 & $110-170$ & 15.80 & 14.94 & $\mathrm{Mn}\left(\mathrm{N}_{2} \mathrm{H}_{3} \mathrm{COO}\right)_{2}$ \\
\hline & $170($ exo) 180 (exo) & 2 & $170-205$ & 29.50 & 27.39 & $\mathrm{MnC}_{2} \mathrm{O}_{4} \cdot \mathrm{N}_{2} \mathrm{H}_{4}$ \\
\hline & $200(\mathrm{ex0})$ & 3 & $205-300$ & 69.90 & $70-55$ & $\mathrm{MnO}$ \\
\hline
\end{tabular}



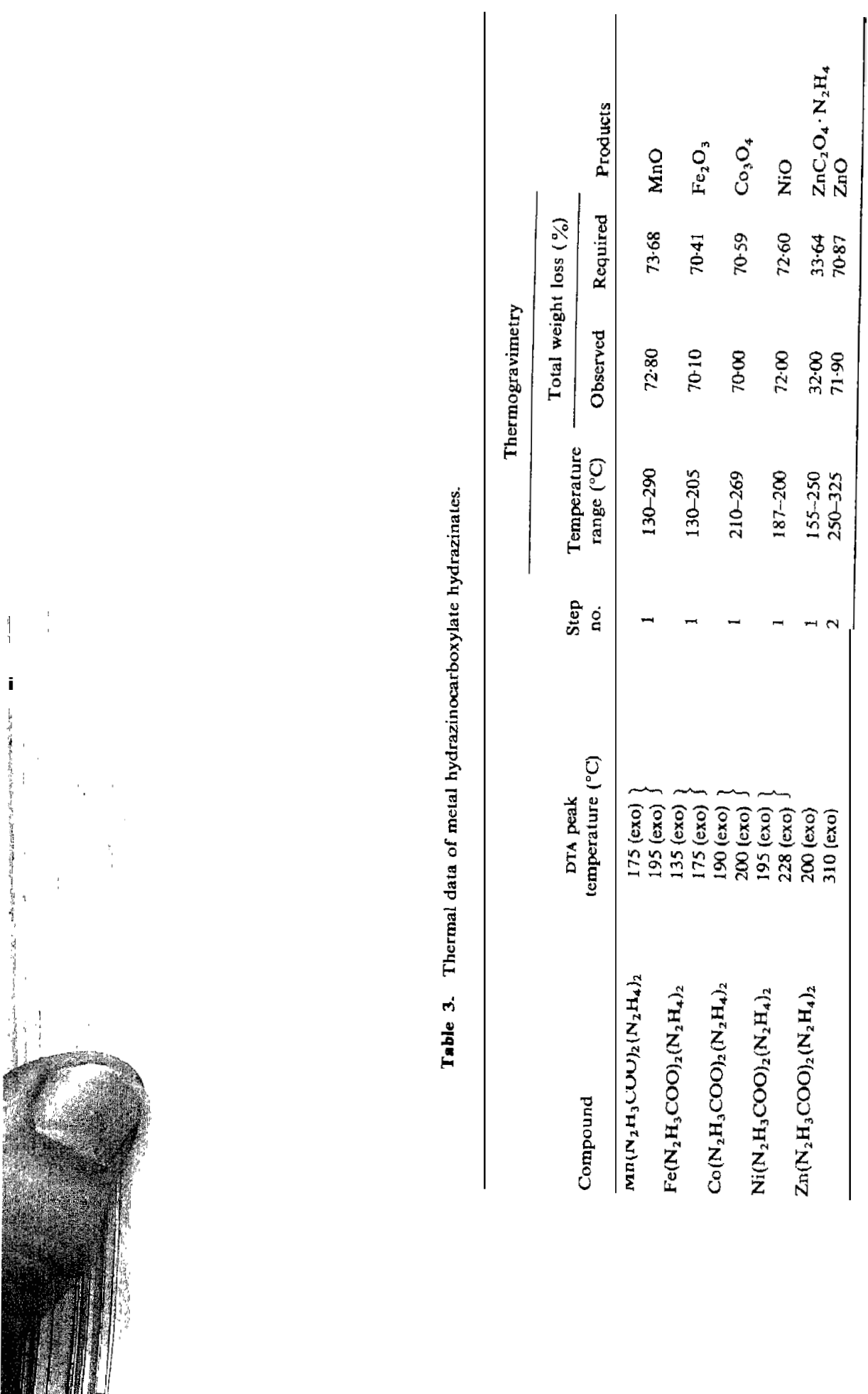
Table 4. Thermal analysis of hydrazinium metal hydrazinocarboxylates.

\begin{tabular}{|c|c|c|c|c|c|c|}
\hline \multirow[b]{3}{*}{ Compound } & \multirow{3}{*}{$\begin{array}{c}\text { DTA peak } \\
\text { temperature }\left({ }^{\circ} \mathrm{C}\right)\end{array}$} & \multirow{3}{*}{$\begin{array}{l}\text { Step } \\
\text { No. }\end{array}$} & \multicolumn{3}{|c|}{ Thermogravimetry } & \multirow[b]{3}{*}{ Products } \\
\hline & & & \multirow{2}{*}{$\begin{array}{l}\text { Temperature } \\
\text { range }\left({ }^{\circ} \mathrm{C}\right)\end{array}$} & \multicolumn{2}{|c|}{ Total weight loss $(\%)$} & \\
\hline & & & & Observed & Required & \\
\hline $\mathrm{N}_{2} \mathrm{H}_{5} \mathrm{Fe}\left(\mathrm{N}_{2} \mathrm{H}_{3} \mathrm{COO}\right)_{3} \cdot \mathrm{H}_{2} \mathrm{O}$ & $\left.\begin{array}{l}165(\mathrm{exo}) \\
180(\mathrm{exo})\end{array}\right\}$ & 1 & $145-220$ & $76-00$ & 75-93 & $\mathrm{Fe}_{2} \mathrm{O}_{3}$ \\
\hline $\begin{array}{l}\mathrm{N}_{2} \mathrm{H}_{5} \mathrm{Co}\left(\mathrm{N}_{2} \mathrm{H}_{3} \mathrm{COO}\right)_{3} \cdot \mathrm{H}_{2} \mathrm{O} \\
\mathrm{N}_{2} \mathrm{H}_{4} \mathrm{Ni}\left(\mathrm{N}_{2} \mathrm{H}_{2} \mathrm{COO}\right)_{2} \cdot \mathrm{H}_{2} \mathrm{O}\end{array}$ & $\begin{array}{l}175(\text { exo) } \\
245(\text { exo) } \\
182 \text { (exo) }\end{array}$ & $\begin{array}{l}1 \\
2 \\
1\end{array}$ & $\begin{array}{l}170-245 \\
245-280 \\
170-210\end{array}$ & $\begin{array}{l}36-50 \\
76 \cdot 10 \\
48 \cdot 00\end{array}$ & $\begin{array}{l}37-61 \\
76-03 \\
46-60\end{array}$ & $\begin{array}{l}\mathrm{CoC}_{2} \mathrm{O}_{4}-2 \mathrm{~N}_{2} \mathrm{H}_{4} \\
\mathrm{CO}_{3} \mathrm{O}_{4} \\
\mathrm{NiC}_{2} \mathrm{O}_{4} \mathrm{~N}_{2} \mathrm{H}_{4}\end{array}$ \\
\hline & $\left.\begin{array}{l}193(\mathrm{exo}) \\
220(\mathrm{exo})\end{array}\right\}$ & 2 & $210-385$ & $76-00$ & 77.67 & $\mathrm{NiO}$ \\
\hline $\mathrm{N}_{2} \mathrm{H}_{5} \mathrm{Zn}\left(\mathrm{N}_{2} \mathrm{H}_{3} \mathrm{COO}\right)_{3} \cdot \mathrm{H}_{2} \mathrm{O}$ & $\begin{array}{l}145 \text { (endo) } \\
195 \text { (exo) }\end{array}$ & $\begin{array}{l}1 \\
2\end{array}$ & $\begin{array}{r}70-165 \\
165-215\end{array}$ & $\begin{array}{l}18 \cdot 00 \\
53-00\end{array}$ & $\begin{array}{l}18.16 \\
55.07\end{array}$ & $\begin{array}{l}\mathrm{Zn}\left(\mathrm{N}_{2} \mathrm{H}_{3} \mathrm{COO}\right)_{2}\left(\mathrm{~N}_{2} \mathrm{H}_{4}\right)_{2} \\
\mathrm{ZnC}_{2} \mathrm{O}_{4}\end{array}$ \\
\hline & 240 (exo) & 3 & $215-390$ & $76-00$ & $76 \cdot 17$ & $\mathrm{ZnO}$ \\
\hline $\mathbf{N}_{2} \mathrm{H}_{5} \mathrm{Mg}\left(\mathrm{N}_{2} \mathrm{H}_{3} \mathrm{COO}\right)_{3}$ & $\begin{array}{l}120 \text { (endo) } \\
320 \text { (exo) }\end{array}$ & $\begin{array}{l}1 \\
2\end{array}$ & $\begin{array}{r}50-135 \\
135-343\end{array}$ & $\begin{array}{l}36-00 \\
72.20\end{array}$ & $\begin{array}{l}37.52 \\
7013\end{array}$ & $\begin{array}{l}\mathrm{MgC}_{2} \mathrm{O}_{4} \cdot 2 \mathrm{~N}_{2} \mathrm{H}_{4} \\
\mathrm{MgCO}_{3}\end{array}$ \\
\hline
\end{tabular}




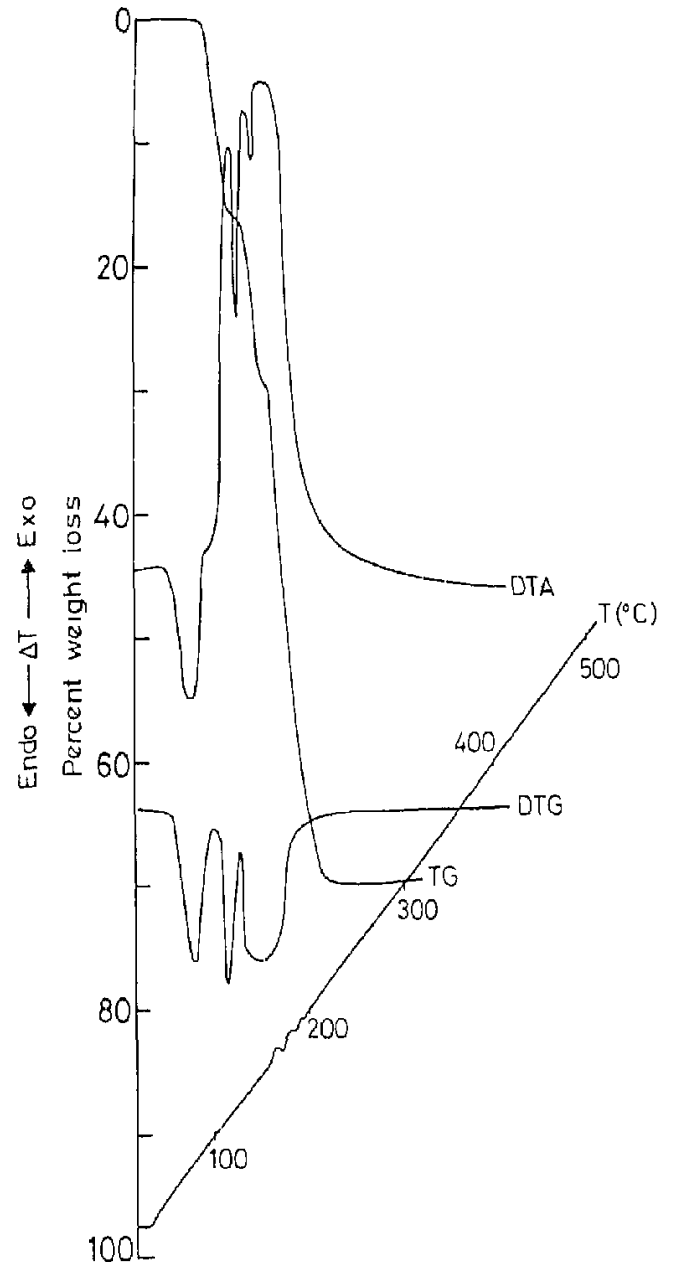

Figure 3. Simultaneous TG-DTG-DTA of $\mathrm{Mn}\left(\mathrm{N}_{2} \mathrm{H}_{3} \mathrm{COO}\right)_{2}\left(\mathrm{H}_{2} \mathrm{O}\right)_{2}$.

the final metal oxides was fixed by the observed weight loss in TG and their characteristic $\mathrm{X}$-ray powder diffraction patterns.

3.4b Metal hydrazinocarboxylate hydrazinates: All the hydrazinate complexes $\left[\mathrm{M}\left(\mathrm{N}_{2} \mathrm{H}_{3} \mathrm{COO}\right)_{2}\left(\mathrm{~N}_{2} \mathrm{H}_{4}\right)_{2}, \mathrm{M}=\mathrm{Mn}, \mathrm{Fe}, \mathrm{Co}, \mathrm{Ni}\right.$ and $\left.\mathrm{Zn}\right]$ except zinc show single step decomposition in TG-DTG. However, DTA shows two exotherms probably corresponding to the decomposition of coordinated hydrazine followed by the decomposition of metal hydrazinocarboxylates to the respective metal oxides. The zinc complex appears to decompose through $\mathrm{ZnC}_{2} \mathrm{O}_{4} \cdot \mathrm{N}_{2} \mathrm{H}_{4}$. The decomposition products (metal oxides)were identified by their $\mathrm{X}$-ray powder diffraction patterns.

3.4c Hydrazinium metal hydruzinocarboxylate derivatives: Thermal decomposition of these complexes $\left[\mathrm{N}_{2} \mathrm{H}_{3} \mathrm{M}\left(\mathrm{N}_{2} \mathrm{H}_{3} \mathrm{COO}\right)_{3} \cdot \mathrm{H}_{2} \mathrm{O}, \mathrm{M}=\mathbf{F e}\right.$, Co, $\mathrm{Ni}$ and $\mathrm{Zn}$, and $\left.\mathrm{N}_{2} \mathrm{H}_{5} \mathrm{Mg}\left(\mathrm{N}_{2} \mathrm{H}_{3} \mathrm{COO}\right)_{3}\right]$ show one to three steps. The iron complex shows a single step in TG-DTG and two exotherms in DTA. Both cobalt and nickel complexes show 2-step 


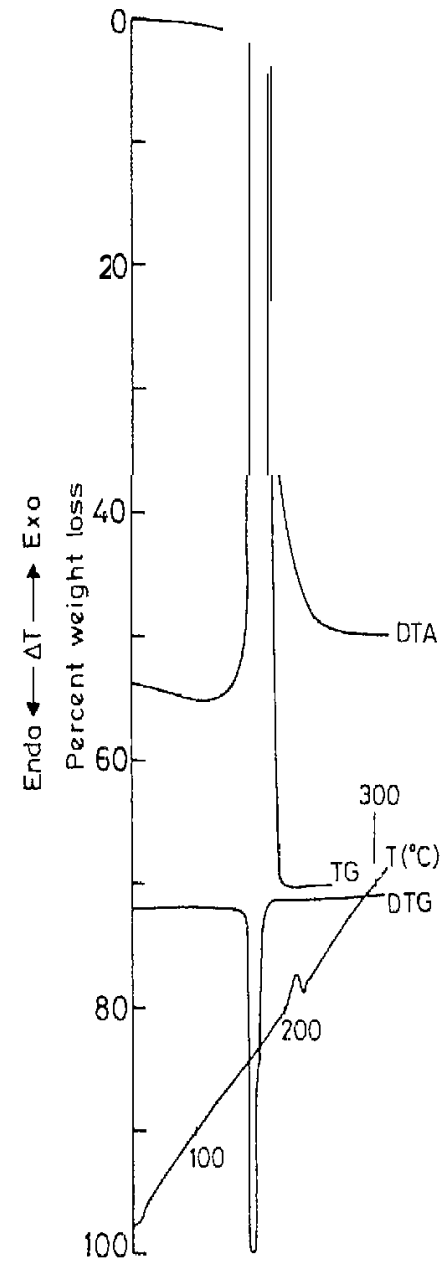

Figure 4. Simultaneous TC-DTG-DTA of $\mathrm{Co}\left(\mathrm{N}_{2} \mathrm{H}_{3} \mathrm{COO}\right)_{2}\left(\mathrm{~N}_{2} \mathrm{H}_{4}\right)_{2}$.

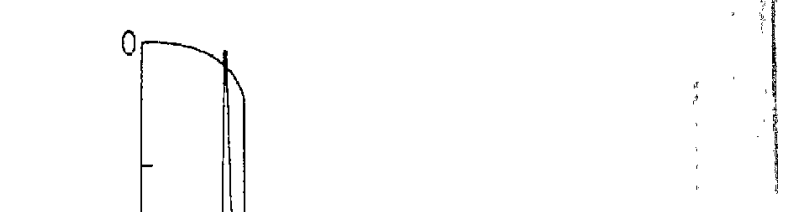

Figure 5. Simultaneous TG-DTG-DT of $\mathrm{N}_{2} \mathrm{H}_{5} \mathrm{Fe}\left(\mathrm{N}_{2} \mathrm{H}_{3} \mathrm{COO}\right)_{3} \cdot \mathrm{H}_{2} \mathrm{O}$.

decomposition in TG.DTG and two exotherms in DTA. The observed weight loss after the first step corresponds to the formation corresponding to the metal oxalate hydrazinate, $\mathrm{MC}_{2} \mathrm{O}_{4} \cdot \mathrm{N}_{2} \mathrm{H}_{4}$ intermediate. The decomposition of zinc and magnesium complexes involve 3-steps each. Possible intermediates could be $\mathrm{M}\left(\mathrm{N}_{2} \mathrm{H}_{3} \mathrm{COO}\right)_{2}\left(\mathrm{~N}_{2} \mathrm{H}_{4}\right)_{2}$, $\mathrm{MC}_{2} \mathrm{O}_{4} \cdot \mathrm{N}_{2} \mathrm{H}_{4}, \mathrm{MC}_{2} \mathrm{O}_{4}$, etc. Final residues of decomposition corresponds to the respective metal oxides as confirmed by x-ray powder diffraction patterns.

\section{Conclusions}

Metal hydrazinocarboxylate derivatives like $\mathrm{M}\left(\mathrm{N}_{2} \mathrm{H}_{3} \mathrm{COO}\right)_{2} \cdot n \mathrm{H}_{2} \mathrm{O}$, $\mathrm{M}\left(\mathrm{N}_{2} \mathrm{H}_{3} \mathrm{COO}\right)_{2}\left(\mathrm{~N}_{2} \mathrm{H}_{4}\right)_{2}$ or $\mathrm{N}_{2} \mathrm{H}_{5} \mathrm{M}\left(\mathrm{N}_{2} \mathrm{H}_{3} \mathrm{COO}\right)_{3} \cdot \mathrm{H}_{2} \mathrm{O}$ are formed by the reaction of metal ions with $\mathrm{N}_{2} \mathrm{H}_{3} \mathrm{COOH}$ in the presence of hydrazine hydrate. The following general observations emerge from the present study. 
(i) None of the metal ions studied form all the three types of hydrazinocarboxylate derivatives.

(ii) Transition metal ions form hydrazinate $\left[\mathrm{M}\left(\mathrm{N}_{2} \mathrm{H}_{3} \mathrm{COO}\right)_{2}\left(\mathrm{~N}_{2} \mathrm{H}_{4}\right)_{2}\right]$ and hydrazinium $\left[\mathrm{N}_{2} \mathrm{H}_{5} \mathrm{M}\left(\mathrm{N}_{2} \mathrm{H}_{3} \mathrm{COO}\right)_{3} \cdot \mathrm{H}_{2} \mathrm{O}\right]$ complexes. Though manganese forms the hydrazinate it does not form the hydrazinium complex.

(iii) Magnesium, calcium and manganese hydrazinocarboxylates form hydrated complexes. Only $\mathrm{Mn}\left(\mathrm{N}_{2} \mathrm{H}_{3} \mathrm{COO}\right)_{2} \cdot 2 \mathrm{H}_{2} \mathrm{O}$ when treated with $\mathrm{N}_{2} \mathrm{H}_{4} \cdot \mathrm{H}_{2} \mathrm{O}$ yields the corresponding hydrazinate, $\mathrm{Mn}\left(\mathrm{N}_{2} \mathrm{H}_{3} \mathrm{COO}\right)_{2}\left(\mathrm{~N}_{2} \mathrm{H}_{4}\right)_{2}$. Magnesium, however, gave anhydrous hydrazinium hydrazinocarboxylate, $\mathrm{N}_{2} \mathrm{H}_{5} \mathrm{Mg}\left(\mathrm{N}_{2} \mathrm{H}_{3} \mathrm{COO}\right)_{3}$.

(iv) Transition metal hydrazinocarboxylate derivatives decompose in air at low temperature $\left(100-200^{\circ} \mathrm{C}\right)$ to the respective metal oxides.

\section{References}

Braibanti A, Bigliardi G, Manotti Lanfredi_AM and Tiripicchio A 1966 Nature 2111175

Braibanti A, Monotti Lanfredi A M, Tiripicchio A and Bigelio F 1967a Acta. Cryst. 23248

Braibanti A, Manotti Lanfredi A M and Tiripicchio A 1967b Z. Krist. 124335

Braibanti A, Dallavalli F, Pellinghelli M A and Leporati E 1968 Inorg. Chem. 71430

Braibanti A, Manotti Lanfredi A M, Pellinghelli M A and Tiripicchio A 1971 Acta. Cryst. 8272261

Ferrari A, Braibanti A, Bigliardi G and Lanfredi A M 1965 Z. krist. 122259

Macek I, Rahten A and Slivnik J 1976Proc. I Eur. Symp. Therm. Anal. (London: Heyden) p. 161.

Patil K C, Soundararajan R and Pai Verneker V R 1979a Proc. Indian Acad. Sci. (Chem. Sci.) 88211

Patil K C, Budkuley J S and Pai Verneker V R 1979b J. Inorg. Nucl. Chem. 41953

Patil K C, Soundararajan R and Goldberg E P 1983 Synth. React. Inorg. Met-Org. Chem. 13(1) 29

Stolle R and Hoffman K 1904 Berichte 374523

Vogel A I 1961 A textbook of quantitative inorganic analysis 3rd ed. (London: Longmans Green) p. 433 